\title{
Influence Analysis of Environmental Information Disclosure Impact on Green Economy Development: An Exploratory Study Based on fsQCA
}

qiang xiong ( $\nabla$ xiongqiang@ujs.edu.cn)

Jiangsu University https://orcid.org/0000-0003-1922-0911

Dan Sun

Jiangsu University

\section{Research Article}

Keywords: Green finance, environmental degradation, fsQCA, patch release, vulnerability severity

Posted Date: September 21st, 2021

DOl: https://doi.org/10.21203/rs.3.rs-771091/v1

License: (c) (1) This work is licensed under a Creative Commons Attribution 4.0 International License.

Read Full License 


\section{Abstract}

Increasing environmental degradation has forced policymakers to include sustainability in the economic growth agenda. Green finance has attracted the attention of policymakers and the industry, but the impact of green finance on social and environmental sustainability has not been confirmed. This study using the panel data of 30 Chinese provinces to investigate the relationship between green finance and environmental degradation. The fuzzy set qualitative comparative analysis (fsQCA) method is utilized for analyzing the mixed effect of green finance on the patch release behavior of $\mathrm{CO}_{2}$ emissions. These factors include vulnerability severity level, attention degree, quantity impact and attack path. The results show that exogenous demand factors including attention and attack type have auxiliary effects when endogenous demand factors including severity and popularity exist as the core antecedent conditions among green finance and environmental degradation. Finally, the policymakers should encourage financial technology to actively participate in environmental protection initiatives that promote green consumption while minimizing the systemic risks caused by financial technology.

\section{Introduction}

For the past few years, the deepening of green financing and a low carbon economy have a great impact on each other and they are very necessary for the betterment of the environment. Low carbon is no longer a problem of the society itself because of Green finance but has become an important part of the environment (Aleksandrov et al. 2013). Green finance and the low carbon economy are closely related concepts, and its essence is the financial sector to make the environmental protection as a basic policy, considering the potential environmental influence when we make the investment and financing decision (Lee 2020). Green finance is a convergence between harmless to the ecosystem conduct and the monetary and business world, be that as it may, not many investigations have connected finance with the environment. Considered the connection between the presentation and social obligation of monetary organizations. Reasoned that natural finance/supportable finance is the best method to decrease ecological debasement. Green finance and low carbons economies are an important part of the national environment (Guild 2020).

Since the start of the mechanical unrest, the monetary area has been an incredible mainstay of human development. The essential job of the worldwide monetary area is to utilize the worldwide reserve funds. Appropriate utilization of speculation empowers improvement in individuals' satisfaction (Criscuolo and Menon 2015). Notwithstanding, due to the breakdown of the monetary framework, individuals have put their investment funds in land bubbles and earth-harming projects, including those that worsen humaninitiated environmental change. Already, the monetary area overlooked the biological system, which empowered the rise or deteriorating of ecological issues, like environment and normal asset exhaustion, environmental change, and contamination (Zhang et al. 2019).

Vulnerability refers to the defects and deficiencies present in the green finance and low carbon during the covid-19 (Iqbal et al. 2021). It is estimated that there may be an average ratio of carbon low in the era of 
covid-19. Tolliver et al. (2020) pointed out that vulnerability discoverers, third-party vulnerability sharing platforms, and research articles must work together to deal with carbon. This is known as collaborative disclosure, which emphasizes that stakeholders such as the government, financial institutes, work together, and cooperate to actively deal with the high risks of carbon. The disclosure of information with government and financial institutes is the most critical node in this collaboration process. The empirical analysis in (Zhang et al. 2021) showed that the instant probability of releasing patches by green finance increased by nearly 2.5 times after the covid- 19 was disclosed. Green finance supports interest in new advances and developments, including environmentally friendly power (). Consequently, we are spurred to inspect the powerful effect of green finance on the carbon dioxide ( $\mathrm{CO} 2)$ outflows of the best ten nations that help green finance.

Until this point in time, hardly any investigations have connected finance to the environment. (Iqbal et al. 2019) recommends that natural manageability can be accomplished through creating financing for sunlight-based energy. A comparative report by (Iqbal et al. 2020) additionally infers that ecological finance/economic finance is the best method to diminish natural corruption. Maintainable finance/green finance empowers interest in new advances and developments, including environmentally friendly power (Wang et al. 2021). Be that as it may, past investigations disregarded the connection between green bonds (an intermediary for green finance) and $\mathrm{CO} 2$ discharges. Green bonds are long-haul monetary instruments in which the returns from green bonds are utilized exclusively to fund projects that are harmless to the ecosystem or decrease contamination in the climate. For instance, green bond incomes are utilized to help sun-powered energy, clean water, and clean vehicle projects.

What is the reason due to which green finance has a great impact on low carbon especially in covid-19? Researchers have worked on many interference factors affecting the low carbon disclosure process. These factors include green finance, green investment and green innovation, and so on. However, the existing research focuses on the "net effect" of single influencing factors, and neither comprehensively identifies the factors influencing the carbon' patch release behavior nor explores the "joint effect" of multiple factors. Especially, the patch release behavior of carbon is a complex situation nowadays and as well as in the future, which cannot be completely explained by the traditional single-factor net effect analysis. This examination makes three commitments to the current writing. In the first place, contrasted with earlier investigations, which for the most part pressure the job of monetary turn of events, rather than just the impact of green finance on natural factors, this investigation presents a spearheading assessment of green finance and $\mathrm{CO} 2$ outflows. Besides, this investigation utilizes the QCA approach that catches the heterogeneous and deviated connection between green finance and low carbon economies. Thirdly, this is one of the principal studies to consider the ten most progressive economies in which green finance has been utilized fundamentally. The exact discoveries on the effect of green finance on their comparing $\mathrm{CO} 2$ emanations go about as benchmarks for different nations. At last, our observational examination gives new experiences into the lopsided reaction of $\mathrm{CO}_{2}$ discharges to green finance use at various QCA. 
Moreover, the interaction among various factors may replace or complement one another. Therefore, which factors affect the carbon's patch release behavior? This is exactly the problem that this paper will explore.

\section{Literature Review And Model Construction 2.1 Theoretical Basis}

According to the classical Synergetic, "synergy" signifies the cooperation between multiple subjects based on common goals, and carbon synergy is a typical synergy theory in environmental science. Finance assumes an essential part in the anthropogenic (i.e., human effect on the climate), yet very little has been done to fuse natural issues into finance. In the course of recent years, the monetary area has focused on green ventures, in this way progressing manageable development. As indicated by (Chen et al. 2021), green monetary instruments can assist with accomplishing a green climate. All the while, monetary delegates, and markets have planned monetary instruments, like green securities, green home loans, green advances for business structures, natural home value programs, "practice environmental awareness" vehicle advances, independent venture organization express advances, and environment charge cards. Likewise, Australia dispatched its first natural store drive, which comprises of medium to long haul finance instruments, which not just finance harmless to the ecosystem undertakings and business exercises yet in addition support feasible turn of events and environment-related ventures straightforwardly (NATF, 2019). Important components of a collaborative environment include green finance, green innovation, the green investment that was the major cause of low carbon economies (Nawaz et al. 2021). During the process of carbon synergy of the green finance release behavior, it can be observed that the vulnerability information disclosure significantly affects the environment. Therefore, under the influence of external environmental factors, information subjects such as green financing have a great influence on low carbon.

\subsection{Model construction}

Vulnerabilities exist objectively in green finance, which is not threatening in itself. However, vulnerabilities can cause damage if they are discovered and low green financing uses them to carry out attacks. The main way to mitigate green finance and low carbon is by developing patches. The synergy demand of vulnerability patch release emanates from two aspects: endogenous requirements formed by the vulnerability itself and exogenous requirements formed by the external security environment (Nassani et al. 2017). In this paper, the main factors of endogenous demand that are considered include severity and popularity, and the main factors of exogenous demand that are considered include the attack type and attention degree. Both internal and external demands reflect the harmful degree of green finance. The so-called harm degree refers to the loss of criticality caused to the environment's information assets when the vulnerabilities are exploited (Geddes et al. 2020). It is mainly related to the severity of loss and the possibility that the vulnerabilities will be exploited, i.e., 


\section{Criticality $=$ Severity $\times$ Probability}

In the above formula, "Severity" refers to the severity of the direct impact on system security when the vulnerability is exploited. Furthermore, "Probability" is availability, which refers to the possibility of software vulnerability being exploited. In this paper, "Severity" mainly refers to the severity of vulnerabilities, while "Probability" is mainly related to the popularity, attack types, and attention degree. All variables are defined below.

\section{(1) Vulnerability severity level}

The severity of software vulnerability reflects the impact of green finance: A higher severity of the vulnerability signifies a higher vulnerability of the low carbon. Ren et al. (2020) studied green finance and the loss of carbon in terms of the return-on-investment model after being hacked. The authors concluded that under a given potential loss level, green finance significantly affects the environmental decisionmaking behavior. Due to the existence of deterrence effect. A higher severity level of vulnerabilities signifies a higher probability of success of low carbon. Therefore, the higher severity of green finance vulnerabilities leads to a higher possibility of low carbon developing their patches.

\section{(2) Vulnerability popularity}

In this paper, the popularity of green finance refers to the number of related products affected by low carbon economies. Green finance is more likely to be exploited if it has a high involvement and a wide scope of influence. Jin et al. (2021) pointed out that enterprises tended to be compatible with affiliated enterprises when the high level of green financing. The resulting system vulnerability and commonality increase the popularity of green finance and ultimately affect the overall carbon level. In recent years, the trend of open-source green finance has become the mainstream of financing, and it has evolved into an important part of the investment. However, it poses a major hidden danger for the green investment, and the loopholes existing in open-source green finance may affect low carbon. When a vulnerability may affect a high level of carbon, it will potentially cause high profits to green finance and its investors. Therefore, the vulnerability scope increases in this case and green finance are more likely to develop patches for such vulnerabilities.

\section{(3) Attack path}

The usage authority levels of different green financing are not consistent. According to the user authority level, green financing can be divided into three kinds: green innovation, green investment, and green bond, and their permissions are sorted from lowest to highest. Higher permission required to exploit the vulnerability lowers the vulnerability to be attacked. Banga (2019) explored the security defense strategy of individual organizations through network scope, attack type, loss possibility, and technology cost, and 
found a network attack to be more threatening than a local attack. Saeed Meo and Karim (2021) developed models to assess the unavailability of an information network due to attacks that exploit green financing. The authors concluded that the scope and probability of attack increased due to green finance interconnection.

\section{(4) Attention degree}

The third-party vulnerability sharing platform, whether it is the CNNVD, CNVD, BuTian, or VulBox, etc., will attract the attention of various groups in similar fields, a significant positive correlation exists between green finance and low carbon economies. The higher the media attention of the listed companies, the better the recognition of the social responsibility report issued by them. Based on the diffusion of innovations theory, Ransbotham et al. found that the probability of attack will be greatly reduced if the vulnerability information is disclosed through the environment. However, improper disclosure of vulnerability information will lead to a higher number of green financing. The higher the attention paid to the disclosed vulnerability, the more likely it is to be attacked by carbon and the more likely it is for green financing to develop patches. To sum up, this paper puts forward the following conceptual model of green financing vulnerability patch release, as shown in Figure 1:

\section{Design Of Research}

\subsection{Selection of research methods}

The Qualitative Comparative Analysis (QCA) method adopted in this paper is a case-oriented method instead of a variable-oriented research method. QCA has been applied comprehensively in organization and management research at the technical analysis and research method levels, and the fuzzy set qualitative comparative analysis (fsQCA) method has superior performance for studying "joint effect" and "interactive relationship" (Elliott 2013). Therefore, this paper uses the fsQCA method to analyze the "joint effect" of various factors on patch release behavior and the "interactive relationship" among various factors to identify the single factors influencing the carbon economies ' and green finance. The analysis results in a summary of the combination of factors that affect green finance in low carbon economies.

\subsection{Selection and measurement of variables}

According to the above analysis, the choice of releasing or not releasing the patches is made as a result of the low carbon effect in the model construction, i.e., the dependent variable. Green finance, green innovations are considered as independent variables that reflect the internal and external factors of carbon and measure the harm degree of vulnerabilities. This study uses the panel data for thirty Chinese provinces from 2003 to 2017 . All the Data were collected from the National Bureau of Statistics of China. Table 1 shows the description of each variable 


\section{Name of Description of variables variables}

Iow If the carbon economies have released the vulnerability patch, the value is 1 ; Otherwise, carbon it is 0

economies

green Green finance has a direct impact on carbon economies. If the green financing is high finance the ratio of the carbon in the environment is low and if the green financing is low then the ratio of carbon in the environment is high. It takes a value in the range $[0,10]$

green Green innovation has a direct impact on carbon economies. If the green innovation is innovation high the ratio of the carbon in the environment is low and if the green innovation is low then the ratio of carbon in the environment is high.

\section{Results And Discussion}

\subsection{Descriptive statistics}

A descriptive statistical analysis of antecedent and outcome variables involved in the study is carried out, and the basic results are provided in Table 2. It can be noted from Table 2 that the correlation among attention degree, attack type, severity, and popularity is not very strong among low carbon, green finance, and green innovation. But without considering other factors, these four antecedents have a positive correlation with patch release. The paper analyzes the data further based on this information.

Table 2

Correlation analysis

\begin{tabular}{|c|c|c|c|c|c|c|c|}
\hline & $\begin{array}{l}\text { Average } \\
\text { Value }\end{array}$ & $\begin{array}{l}\text { Standard } \\
\text { Deviation }\end{array}$ & $\begin{array}{l}\text { Attention } \\
\text { Degree }\end{array}$ & $\begin{array}{l}\text { Attack } \\
\text { Type }\end{array}$ & Severity & Popularity & $\begin{array}{l}\text { Patch } \\
\text { Release }\end{array}$ \\
\hline low carbon & 822.630 & 435.0967 & 1 & & & & \\
\hline $\begin{array}{l}\text { green } \\
\text { finance }\end{array}$ & 0.846 & 0.3611 & 0.03 & 1 & & & \\
\hline $\begin{array}{l}\text { green } \\
\text { innovation }\end{array}$ & 5.923 & 1.8925 & 0.092 & $0.248^{*}$ & 1 & & \\
\hline
\end{tabular}

\subsection{Calibration of variables}

The key difference between fuzzy sets and conventional variables lies in the way they are conceptualized and labeled. The condition and result data must be calibrated before the QCA is executed. It is necessary to specify a target set in order to calibrate it as a fuzzy set, which not only constitutes the calibration of the set but also provides a direct connection between the theoretical discourse and empirical analysis. 
In this study, the fsQCA was adopted, and the related antecedents and results were calibrated as fuzzy set membership scores using the method of direct calibration. Set membership does not have to be binary $(0 / 1)$. Rather, in fsQCA, the aim is to calibrate set membership in such a way that levels of membership represent meaningful groupings. Out of the scores, the intersection point value has the greatest fuzziness, which determines whether most cases belong to or not belong to the target set on the value of the fixeddistance scale variable. Among the antecedent variables related to green finance and $\mathrm{CO} 2$ emissions chosen in this paper, the mean is utilized as the calibration standard for the intersection of attention degree, severity, popularity, and attack type. Other calibration standards are "mean - standard deviation" and "mean + standard deviation". The reason for this choice is that the mean reflects the average level of all disclosed software vulnerabilities, while the standard deviation reflects the difference of disclosed software vulnerabilities in a certain index. Table 3 shows the final calibration results.

Table 3

Calibration threshold of each variable

\begin{tabular}{|llll|}
\hline Variables & $\begin{array}{l}\text { Nonmembership anchor } \\
\text { point }\end{array}$ & $\begin{array}{l}\text { Intermediate anchor } \\
\text { point }\end{array}$ & $\begin{array}{l}\text { Complete membership anchor } \\
\text { point }\end{array}$ \\
\hline $\begin{array}{l}\text { Attention } \\
\text { degree }\end{array}$ & 387.53 & 822.63 & 1257.73 \\
\hline Severity & 4.03 & 5.923 & 7.82 \\
\hline Popularity & 1.000 & 3 & 5 \\
\hline Attack type & 0 & - & 1 \\
\hline
\end{tabular}

\subsection{Single-factor necessity analysis}

Based on the general steps of the fSQCA, this paper first checks whether a single factor and its non-set constitute a necessary condition for the results, i.e., patch release and patch non-release among the green finance and $\mathrm{CO} 2$ emissions. This means that it is verified whether the result set is a subset of the single factor and its non-set, which is usually measured by consistency. It is determined that the single factor or non-set is the necessary condition for the result set when the consistency level is higher than 0.9. Table 4shows the results of fsQCA. It can be observed that all antecedents cannot constitute the necessary conditions for the realization of specific results. The necessity of all single antecedents and their non-sets affecting patch release does not exceed 0.8 , and the necessity of influencing patch non-release does not exceed 0.3. Therefore, all single antecedents do not constitute necessary conditions for patch release or patch non-release. 
Table 4

Test of adequacy and necessity of antecedents

\begin{tabular}{|lllll|}
\hline & Patch release & \multicolumn{3}{c|}{ Non-release } \\
\cline { 2 - 5 } & Consistency & Coverage & Consistency & Coverage \\
\hline Attention degree & 0.439415 & 0.728098 & 0.467467 & 0.271902 \\
\hline Attention degree & 0.560585 & 0.749925 & 0.532533 & 0.250075 \\
\hline Severity & 0.462026 & 0.707507 & 0.544134 & 0.292494 \\
$\sim$ Severity & 0.537975 & 0.770739 & 0.455867 & 0.229261 \\
\hline Popularity & 0.410509 & 0.721806 & 0.450713 & 0.278192 \\
\hline$\sim$ Popularity & 0.589491 & 0.753528 & 0.549287 & 0.246473 \\
\hline Attack Type & 0.613569 & 0.753623 & 0.571429 & 0.246377 \\
\hline$\sim$ Attack Type & 0.386431 & 0.719780 & 0.428571 & 0.280220 \\
\hline
\end{tabular}

\section{Analysis of the adequacy of antecedent configuration}

Antecedent configuration analysis tries to reveal the sufficiency of the outcome caused by different configurations composed of multiple antecedent conditions. In this study, the fSQCA 3.0 software was used to process data for constructing the truth table: $\triangle$ Case frequency threshold. Rihoux et al. proposed choosing the frequency threshold such that the number of retained cases is higher than or equal to $75 \%$ of the total number of cases. In this paper, the total number of cases is 916 . Four antecedent conditions will generate 16 configurations in the truth table. So the frequency threshold of the effective antecedent condition combination is set to 50 .

\Raw consistency threshold. Consistency measures "how closely a perfect subset relation [between a configuration and an outcome] is approximated"; in the simple case of crisp sets, consistency is the proportion of cases exhibiting the configuration that exhibit the outcome. In this paper the outcome is path-release. It is good practice to establish different consistency thresholds for necessity and sufficiency analyses and to not interpret subset relations that do not meet these thresholds. The antecedent condition configuration where the raw consistency value is higher than the threshold is a subset of the outcome, and the outcome is assigned 1; otherwise, it is 0 . In this paper, the minimum threshold of raw consistency is set to 0.75 .

Three kinds of solutions are obtained after analyzing the truth table: complex solution, concise solution, and optimized solution. The complex solution does not include any logical remainder. The intermediate solution only includes the logical remainder in line with the theoretical direction and empirical evidence. And the simplified solution includes all the logical remainder without evaluating its rationality. So intermediate solution is considered to be the first choice for reporting and interpretation in QCA results. 
Referring to the existing research, this paper reports the intermediate solution, and the simplified solution is used as an auxiliary. Table 5 shows the configuration results of software vendors' conditions before the patch release, where " $\odot$ " and " $\otimes$ " indicate the existence and absence of core antecedent condition, respectively, and "๑" and " $\otimes$ " indicate the existence and absence of an auxiliary antecedent condition, respectively. Blanks can indicate either existence or absence of antecedent conditions.

Table 5 shows that the consistency of each configuration and the total consistency are higher than the minimum acceptable standard of 0.75 in both models. The total coverage rates of patch release and nonrelease are 0.443 and 0.416 , respectively, which are equal to those obtained using QCA research in the fields of organization and management. It can be gathered from the results that the fsQCA effectively identifies six antecedent configurations. The identifications can indicate whether the existence or absence of antecedent factors has a positive or negative impact on the patch release decision of the software vendors.

Table 5

Pre-condition configuration for patch release or patch non-release

\begin{tabular}{|lllllll|}
\hline Type & \multicolumn{3}{l}{ Patch release } & \multicolumn{4}{c|}{ Non-release } \\
\cline { 2 - 7 } & D1 & D2 & D3 & N1 & N2 & N3 \\
\hline Attack type & $\bullet$ & & $\bullet$ & $\otimes$ & $\bullet$ & $\bullet$ \\
Attention degree & & $\bullet$ & $\otimes$ & $\bullet$ & & $\otimes$ \\
\hline Severity & $\bullet$ & $\bullet$ & & $\otimes$ & $\otimes$ & \\
Popularity & $\otimes$ & $\otimes$ & $\bullet$ & & $\otimes$ & $\otimes$ \\
Coverage rate & 0.138 & 0.227 & 0.234 & 0.167 & 0.21 & 0.179 \\
\hline Net coverage rate & 0.138 & 0.071 & 0.078 & 0.07 & 0.07 & 0.137 \\
\hline Consistency & 0.791 & 0.8 & 0.784 & 0.756 & 0.821 & 0.721 \\
\hline Total coverage & 0.443 & & & 0.416 & & \\
\hline Total consistency & 0.788 & & & 0.77 & & \\
\hline
\end{tabular}

In patch release implementation configurations D1 (attack type + severity $+\sim$ popularity) and D2 (attention degree + severity $+\sim$ popularity), the severity of vulnerability exists as the core precondition, and the lack of popularity plays an auxiliary role. In the former configuration, the attack type plays an auxiliary role, while in the latter configuration, attention plays a core role when the attack type either exists or is absent. The attack type and popularity play a core role, while the lack of attention degree plays an auxiliary role in D3 (attack type $+\sim$ attention degree + popularity).

The vulnerability severity and the lack of attack type are the core antecedents, and the attention rate plays a key role in configuration $\mathrm{N} 1$ ( attack type + attention degree $+\sim$ severity), which causes software 
vendors not to release patches. In N2 (attack type + severity + popularity), the vulnerability severity and the lack of popularity are core antecedents, and the attack type exists as auxiliary antecedents. In N3 (attack type $+\sim$ attention $+\sim$ popularity), the popularity and the lack of attention degree are core antecedents, and the attack type exists as core antecedents.

The total consistency of the configurations in this study is 0.788 , which indicates that the interpretation degree of the six configurations concerning the patch release behavior of software vendors is $78.8 \%$. The total coverage rate is 0.701 , which indicates that the research results can cover $70.1 \%$ of cases. It is necessary to simultaneously analyze the consistency and coverage of all configurations during the process of qualitative comparative analysis. The consistency of the six configurations is about 0.79 , which proves that there is a good subset relationship between the six configurations and the patch release of software vendors, signifying a high explanatory capability of the patch release behavior. It can be concluded based on the results that the fsQCA can effectively identify six antecedent configurations, which show how the existence or absence of each element in different antecedent configurations affects the release behavior of software vendors' patches.

\subsection{Configuration effect as a Robustness test}

Based on well-known research results, this paper adjusts the consistency threshold and reprocesses the sample data. The original minimum consistency threshold is adjusted from 0.75 to 0.76 . The antecedent configuration obtained under the consistency threshold of 0.76 is the same as that obtained under the consistency threshold of 0.75 , which is consistent with the above-mentioned conclusion. Therefore, this paper obtains robust research conclusions.

This paper puts forward the following three research propositions, based on the antecedent configuration of green finance' patch release and the theoretical analysis behind it, and comparison with the antecedent configuration of patch release:

(1) The severity of vulnerabilities in the core prerequisite for low carbon to develop patches under the condition of the environment. The comparative analysis of vulnerability severity shows that the vulnerability with a high severity leads to a relatively high proportion of patch release sample cases (D1, D2). The coverage rates for these two cases are 0.138 and 0.227 , respectively, and the consistency rates are 0.791 and 0.8 , respectively, which are higher than the total consistency of patch release sample cases of 0.788 . However, the proportion of sample cases with low severity vulnerabilities $(\mathrm{N} 1, \mathrm{~N} 2)$ that causes the patches to be not released is also high, with coverage rates of 0.167 and 0.21 , and consistency rates of 0.756 and 0.821 , respectively. Cremonini and Nizovtsev pointed out that when the attackers had complete information about green finance vulnerabilities, and could choose between different attack targets to carry out low carbon. Arora et al. [4] proved through empirical analysis that high vulnerabilities have a high impact on low carbon' patch release behavior. All of these results demonstrate theoretically that the severity of vulnerabilities is one of the core elements in the patch release of low carbon. 
However, only a single core condition of a high-risk level is not enough to force low carbon to release pathed. In D1, the attack type exists as an auxiliary condition, i.e., it enlarges the possibility of the vulnerability being attacked in a network remote attack. In D2, attention exists as another core condition. Higher attention causes a higher loss of social reputation caused by not releasing the patches in time. Therefore, the reasons for the patch release rates of ultra-high-risk and high-risk vulnerabilities being only $73.75 \%$ and $75.87 \%$ among the vulnerabilities disclosed in CNVD in 2019 can be inferred: High-risk vulnerabilities that have not been patched are not paid enough attention, or the attack types are mainly non-remote network attacks.

\section{Proposition 1}

In the collaborative disclosure of software vulnerability patched by software vendors, one of the necessary conditions for software vendors to develop patches is the severity of endogenous demand factors. However, it is not a sufficient condition. The types of vulnerability attacks and attention of exogenous demand factors jointly affect the decision of software vendors to release vulnerability patches.

(2) Analysis of configuration D3 shows that the coverage rate of the configuration is the highest among all configurations at 0.234 . Comparing it with configurations $\mathrm{N} 2$ and N3, if the antecedent of network attack exists and if the antecedent of popularity does not exist, it is very likely that software vendors will not release patches irrespective of whether the attention and severity are missing or pending, e.g., the coverage rates of N2 and N3 are 0.21 and 0.179 , respectively. Hacker attacks can be divided into direct attacks and derivative attacks through the network. Huang and Behara used the network theory to study the impact of targeted and random attacks on enterprise security investment. The authors pointed out that in case of multiple interrelated information systems and security incidents that can cause heavy losses, enterprises should pay attention to hacker network intrusion. On the other hand, for software vendors, the disclosure of vulnerability information positively impacts the market value of enterprises: the higher the popularity, the greater the impact. Generally speaking, the number of products affected by the vulnerabilities in open source software is higher than that in closed commercial software. However, both software users and research institutions prefer the former. The reason for this preference is due to its openness and the convenience of remedying the vulnerabilities at any time. Empirical analysis results such as those presented by Arora in showed that open source suppliers released patches faster than closed source suppliers. At the same time, a higher number and variety of products that are affected decreased the marginal cost of developing patches for each product, and increased the enthusiasm of software vendors to release patches.

\section{Proposition 2}

When the exogenous demand factor is a remote network attack, it is easier for the vulnerability with high endogenous demand factor to get a patch, and its software security is highly reliable. Vulnerable 
software vendors with low coverage are less motivated to develop patches, and their security is relatively unreliable.

(3) During the process of software vulnerability patch management, the vulnerability severity should coexist as a core condition of vulnerability attention is a core precondition. This increases the possibility of the software vendors issuing patches. If attention exists as the core condition but vulnerability severity is missing, and the attack type is missing as the auxiliary antecedent condition, the software manufacturer's willingness to develop vulnerability patches declines irrespective of the extent of the vulnerability, i.e., N1 is configured in the table with a coverage rate of 0.167 and a consistency of 0.756 . Many disclosed vulnerabilities have a high attention rate but do not present a significant level of threat. For this type of vulnerability, green finance institutions are unlikely to carry out research and development of their products. For example, CNVD-2020-20443 is a local denial-of-service vulnerability present in FlashFXP, which can be exploited by attackers to cause denial-of-service attacks. It is of great concern to users, with an attention rate of 1136 and a hazard level of 4.9. However, software vendors have not released a patch for this vulnerability as it presents a moderate risk and the attack type is local.

\section{Proposition 3}

In the collaborative disclosure of software vulnerability patches, one of the necessary conditions is high attention to exogenous demand factor vulnerabilities. However, it is not a sufficient antecedent. If the vulnerability is low in severity and mainly suffers from non-remote network attacks, software vendors may consider giving up patch development regardless of vulnerability attention.

\section{Conclusion And Policy Implication}

It is neither technically nor economically feasible to ensure absolute security of the network for green finance. Vulnerability disclosure is the central link of information security for green financing. To deal with the current low patch repair rate of climate change, this paper used fSQCA to capture sample case data of green finance and carbon emissions It discussed the "joint effect" of vulnerability attention, severity, popularity and attack type of green finance on carbon emissions, in order to explore the factor configuration under different circumstances. It was observed that the antecedents were neither a necessary nor a sufficient condition for green finance. $\mathrm{CO}_{2}$ emissions vendors' patch release behavior was the result of multiple antecedents, which was characterized by "multiple concurrency". This paper analyzed the antecedent configuration of software vendors' patch release and patch non-release and obtained three main paths to promote software vendors to release patches, where each path was made up of many antecedent elements. This conclusion shows that, unlike previous studies that focused on a single factor, such as vulnerability harm degree, patch cost, and benefit, etc., the software vendors' patch release behavior should be examined from an overall perspective. This fully explains why nearly $30 \%$ of 
the ultra-high-risk, high-risk, medium-threat and low-risk vulnerabilities published by the China National Vulnerability Database of Information Security cannot be effectively patched. Therefore, the work presented in this paper has extended the research on software vulnerability patch management to a certain extent from a single perspective to an overall system perspective.

China Green Finance Association Convention on disclosure and disposal of vulnerability information stipulates that "green finance institutions must actively respond to the vulnerability information provided by the government, vulnerability platform and vulnerability submitter, verify and confirm in time and provide and release vulnerability solutions." Performing effective vulnerability green finance management is the foundation against environmental pollution. Based on the aforementioned research conclusions, it is suggested that attention should be paid to the following factors during the process of vulnerability management:

(1) The accuracy of vulnerability sharing information of financial institutions is the basis of a successful follow-up. A standardized vulnerability information receiving, processing, and publishing process should be established to ensure the authenticity and integrity of vulnerability information. The information submitted by the vulnerability submitters should be pre-verified, such as the possible ways of attack and the attention paid by environmental pollution-related stakeholders to the vulnerability. Based on the repair rules and required cycles of different types of vulnerabilities, flexible and practical vulnerability public disclosure time should be fully studied and formulated. Especially, the accurate evaluation of endogenous demand factors of vulnerabilities significantly influences the software vendors' decisions regarding patch repair.

The dynamic changes should be grasped through relevant technical means for monitoring the external demand indicators. When the exogenous demand conditions of vulnerabilities with high endogenous demand factors reach a certain level, software vendors will actively develop patches with high probability. In this case, there is no need to adjust the protection period of third-party platforms for vulnerability disclosure. We can focus on the vulnerabilities with low endogenous demand characteristics, such as severity and popularity, and take measures to encourage software vendors to develop patches.

(2) Regular communication should be maintained with vulnerability discoverers. The vulnerability information related to green finance institutions should be discovered in time and responded positively. The vulnerability severity, popularity, attack type, and social concerns should be verified, and active cooperation should be maintained with collaborative management between green product development and release in the market. The vulnerability patches or solutions should be provided, in order to strive for a longer vulnerability protection period and low patch development costs. Technical and service preparations should be made for remote product upgrades and user system maintenance to ensure the effectiveness, timeliness, and coverage of vulnerability repair measures.

There are still a few shortcomings in this study: 1) the identification of factors influencing the carbon patch release behavior is not comprehensive enough, and 2) the research model may not include all antecedents. For example, competition factors among enterprises, Carbon cost and responsibility sharing 
of vulnerability loss may affect the patch release behavior. To deal with this issue, more comprehensive data will be collected for further exploration.

\section{Declarations}

\section{Ethics approval:}

Ethical approval was not required for this research.

\section{Consent to participate:}

Not applicable.

\section{Consent for publication:}

Not applicable.

\section{Author contribution:}

Qiang Xiong was major contributor in writing the manuscript. Dan Sun analyzed the data. All authors read and approved the final manuscript. All authors of this manuscript have directly participated in the planning, execution, and analyses of this study.

\section{Funding:}

No Funding.

\section{Competing interests:}

The authors declare no competing interests.

\section{Availability of data and materials:}

All materials and data which was generated or analyzed during this study were included in this article.

\section{References}

1. Aleksandrov N, Espinoza R, Gyurkó L (2013) Optimal oil production and the world supply of oil. J Econ Dyn Control 37:1248-1263. https://doi.org/10.1016/j.jedc.2013.01.015 
2. Banga J (2019) The green bond market: a potential source of climate finance for developing countries. J Sustain Financ Invest 9:17-32. https://doi.org/10.1080/20430795.2018.1498617

3. Chen Q, Ning B, Pan Y, Xiao J (2021) Green finance and outward foreign direct investment: evidence from a quasi-natural experiment of green insurance in China. Asia Pacific J Manag 1-26. https://doi.org/10.1007/s10490-020-09750-w

4. Criscuolo C, Menon C (2015) Environmental policies and risk finance in the green sector: Crosscountry evidence. Energy Policy. https://doi.org/10.1016/j.enpol.2015.03.023

5. Elliott TA (2013) Fuzzy set qualitative comparative analysis: An introduction. Res Notes 1-6

6. Geddes A, Schmid N, Schmidt TS, Steffen B (2020) The politics of climate finance: Consensus and partisanship in designing green state investment banks in the United Kingdom and Australia. Energy Res Soc Sci 69:101583. https://doi.org/https://doi.org/10.1016/j.erss.2020.101583

7. Guild J (2020) The political and institutional constraints on green finance in Indonesia. J Sustain Financ Invest 10:157-170. https://doi.org/10.1080/20430795.2019.1706312

8. Iqbal W, Altalbe A, Fatima A et al (2019) A DEA approach for assessing the energy, environmental and economic performance of top 20 industrial countries. Processes 7:. https://doi.org/10.3390/PR7120902

9. Iqbal W, Fatima A, Yumei $\mathrm{H}$ et al (2020) Oil supply risk and affecting parameters associated with oil supplementation and disruption. J Clean Prod 255.. https://doi.org/10.1016/j.jclepro.2020.120187

10. Iqbal W, Tang YM, Chau KY et al (2021) Nexus between air pollution and NCOV-2019 in China: Application of negative binomial regression analysis. Process Saf Environ Prot 150:. https://doi.org/10.1016/j.psep.2021.04.039

11. Jin $Y, G a o X$, Wang M (2021) The financing efficiency of listed energy conservation and environmental protection firms: Evidence and implications for green finance in China. Energy Policy. https://doi.org/10.1016/j.enpol.2021.112254

12. Lee JW (2020) Green finance and sustainable development goals: The case of China. J Asian Financ Econ Bus. https://doi.org/10.13106/jafeb.2020.vol7.no7.577

13. Nassani AA, Aldakhil AM, Qazi Abro MM, Zaman K (2017) Environmental Kuznets curve among BRICS countries: Spot lightening finance, transport, energy and growth factors. J Clean Prod 154:474-487. https://doi.org/10.1016/j.jclepro.2017.04.025

14. Nawaz MA, Seshadri U, Kumar $P$ et al (2021) Nexus between green finance and climate change mitigation in $\mathrm{N}-11$ and BRICS countries: empirical estimation through difference in differences (DID) approach. Environ Sci Pollut Res 28:6504-6519. https://doi.org/10.1007/s11356-020-10920-y

15. Ren X, Shao Q, Zhong R (2020) Nexus between green finance, non-fossil energy use, and carbon intensity: Empirical evidence from China based on a vector error correction model. J Clean Prod 277:122844. https://doi.org/10.1016/j.jclepro.2020.122844

16. Saeed Meo M, Karim MZA (2021) The role of green finance in reducing CO2 emissions: An empirical analysis. Borsa Istanbul Rev. https://doi.org/https://doi.org/10.1016/j.bir.2021.03.002 
17. Tolliver C, Keeley AR, Managi S (2020) Policy targets behind green bonds for renewable energy: Do climate commitments matter? Technol Forecast Soc Change 157:120051. https://doi.org/10.1016/j.techfore.2020.120051

18. Wang M, Li X, Wang S (2021) Discovering research trends and opportunities of green finance and energy policy: A data-driven scientometric analysis. Energy Policy 154:112295. https://doi.org/10.1016/j.enpol.2021.112295

19. Zhang D, Mohsin M, Rasheed AK et al (2021) Public spending and green economic growth in BRI region: Mediating role of green finance. Energy Policy. https://doi.org/10.1016/j.enpol.2021.112256

20. Zhang D, Zhang Z, Managi S (2019) A bibliometric analysis on green finance: Current status, development, and future directions. Financ Res Lett. https://doi.org/10.1016/j.frl.2019.02.003

\section{Figures}

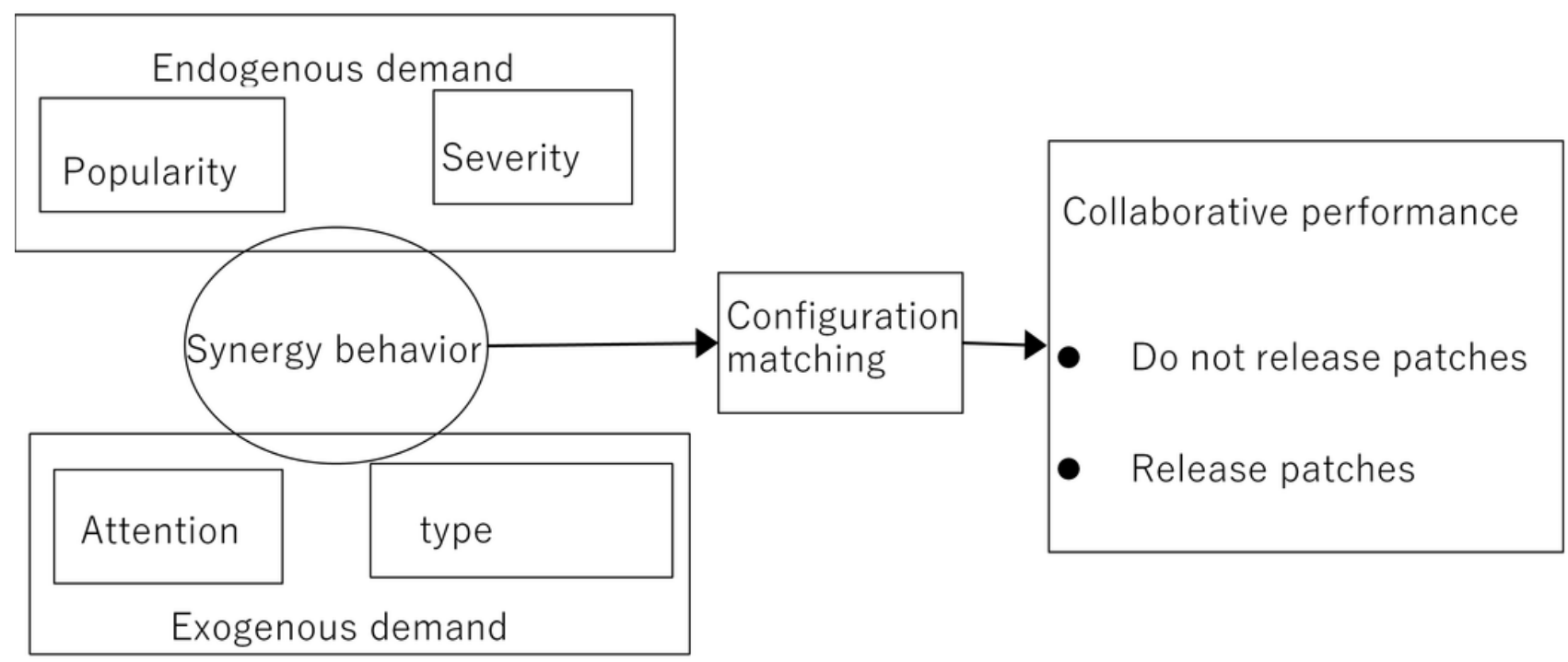

Figure 1

Conceptual model of software vulnerability patch release for green financing 